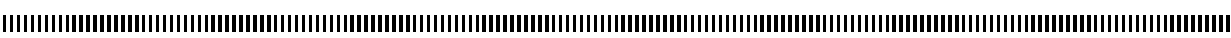

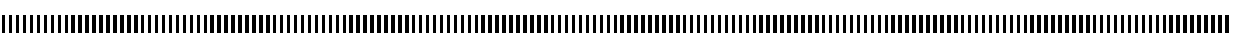
|

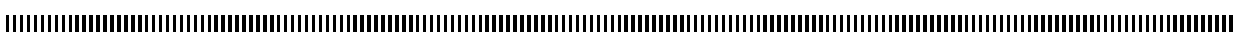
|

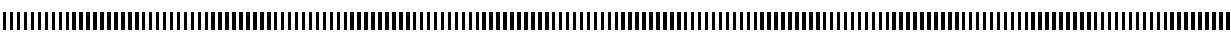
|

\title{
Discrétisation en temps par sous-domaine pour un problème d'advection en milieu poreux
}

\author{
Amel Sboui* — Jérôme Jaffré* \\ * INRIA Roquencourt, BP 105, Le Chesnay, France
}

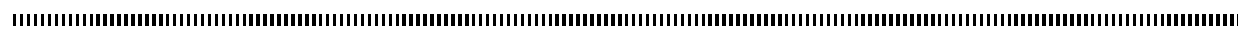

RÉSUMÉ. L'objet de cet article est de présenter une méthode de discrétisation en temps telle que des pas de temps différents sont utilisés dans différents sous-domaines en espace. L'équation d'advection est discrétisée par un schéma décentré amont. Le raccord entre les grilles en temps est réalisé de sorte que le schéma soit conservatif.

ABSTRACT. The aim of this paper is to present a method of time dicretisation which allows the use of different time steps in different subdomains. The advection equation is dicretised by an upwind scheme. The link between the time grids is accomplished in order that the scheme is conservative.

MOTS-CLÉS : Equation d'advection, Volumes finis, Shémas amont, Discrétisation en temps par sousdomaine.

KEYWORDS : Advection equation, Finite volumes, Upwind scheme, Subdomain time stepping.

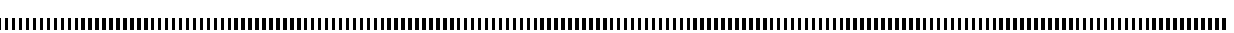

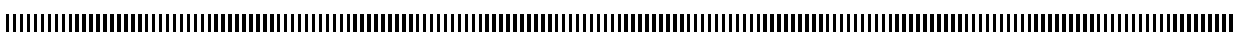
| | |

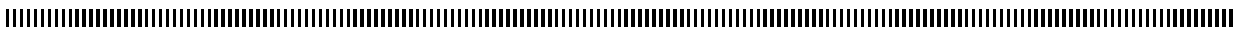




\section{Introduction}

L'étude des écoulements et du transport dans les milieux poreux trouve principalement deux types d'applications, l'hydrogéologie et la simulation de réservoirs d'hydrocarbures. Pour ce qui est des problèmes d'environnement dans le sous-sol, ils concernent principalement les ressources en eau, qui sont aujourd'hui de plus en plus menacées du fait des pollutions ménagères, industrielles et agricoles et d'une consommation intensive. D'autre part le choix de sites de stockage de déchets nécessite de nombreuses études de fiabilité. En particulier le stockage de déchets radioactifs, notamment en couche géologique profonde, est envisagé dans certains pays industrialisés (dont la France) et parfois même déjà mis en œuvre (comme aux Etats-Unis).

L'hydrogéologie est donc un sujet de première importance pour une société comme la nôtre : elle doit pouvoir gérer convenablement les ressources en eau potable et assurer des niveaux de pollution les plus bas possibles à ses habitants. C'est pour ces raisons qu'il est nécessaire de se donner les moyens de prévoir correctement les comportements des écoulements d'eau et le transport de contaminant dans le sous-sol.

Concernant les problèmes de gisements d'hydrocarbures, la volonté constante des industriels d'augmenter les rendements des réservoirs pétroliers, liée en partie à l'épuisement des ressources en hydrocarbures de la planète, nécessite également des efforts importants de modélisation des écoulements de fluide en sous-sol.

Tous ces problèmes conduisent à de nombreux modèles correspondant à diverses situations physiques : écoulements monophasiques, multiphasiques, réactifs, milieux fracturés, modèles de puits, etc. La modélisation numérique joue dans ces domaines un rôle fondamental car les expérimentations en sous-sol sont coûteuses et peuvent ne pas être réalisables. Par exemple, les échelles d'étude pour le transport de contaminants est typiquement de l'ordre de la dizaine de kilomètres voire de la centaine de kilomètres sur des dizaines ou des centaines de milliers d'années. Dans ces conditions, de nombreux problèmes de modélisation et d'ordre purement numérique apparaissent. Notamment, la prise en compte des hétérogénéités dans le sous-sol est une des difficultés principales de l'écoulement et du transport souterrains.

Les hétérogénéités se traduisent par des discontinuités dans les coefficients du système d'équations au passage d'un type de roche à l'autre. Cependant certaines quantités physiques restent continues : la vitesse de Darcy, la pression et les flux de contaminants. Pour un exemple d'une telle situation, citons en particulier [1] concernant les déchets nucléaires.

Les simulations du transport des contaminants dans des situations complexes peuvent conduire à des temps de calcul très importants. Par ailleurs dans des milieux hétérogènes les échelles de temps peuvent varier de façon considérable d'une région à l'autre du domaine de calcul. Afin d'économiser du temps de calcul, nous présentons dans cet article une méthode de discrétisation en temps telle que des pas de temps différents sont utilisés dans différents sous-domaines en espace de sorte qu'ils s'accordent le mieux possible à 
l'échelle de temps du sous-domaine considéré. La discrétisation locale en temps appliquée aux lois de conservation en a déjà été proposée par Stanley Osher et Richard Sanders [10] pour un shéma volumes finis. Plus récemment Clint Dawson et Robert Kirby [4, 11] ont étendu la méthode au cas de schémas d'ordre plus précis que le schéma d'Euler d'ordre 1.

Le couplage de différentes discrétisation en temps a été aussi souvent étudié à partir du point de vue du parallélisme [2] en couplage fluide-structure [6,7]. D'autres techniques de raffinement en temps ont été développées dans [8,3]. Un point de vue décomposition de domaine espace-temps est présenté dans [5]. Nous nous proposons dans cet article de traiter le cas d'un problème d'advection, prototype d'une situation commune en hydrogéologie.

Soit $\Omega$ un ouvert de $\mathbb{R}^{2}$ de frontière $\Gamma=\partial \Omega$ et soit $\vec{n}_{\Gamma}$ la normale extérieure à $\Omega$. On modélise le transport d'un contaminant en milieu poreux par l'équation de conservation.

$$
\begin{aligned}
\Phi \frac{\partial c}{\partial t}+\operatorname{div}(\vec{u} c) & =0 \quad \text { dans } \Omega, \\
c(., t) & =1 \quad \text { sur } \quad \Gamma_{-}=\left\{(x, y) \in \partial \Omega \quad \mid \vec{u} \cdot \vec{n}_{\Gamma}<0\right\}, \\
c(., 0) & =0 \quad \text { dans } \Omega .
\end{aligned}
$$

$c$ est la concentration du contaminant dissous dans l'eau, $\Phi$ la porosité supposée égale à 1 dans la suite, $t \in[0, T]$, et $\Gamma_{-}$représente la frontière amont de $\Omega$ par rapport à $\vec{u}$, la vitesse de Darcy de l'eau. Celle-ci est solution d'un problème d'écoulement résolu auparavant :

$$
\begin{array}{rlrlrl}
\operatorname{div} \vec{u} & =0, & & \vec{u} & =-\frac{K}{\mu} \vec{\nabla} p \\
\vec{u} \cdot \vec{n} & =q_{d} & \text { sur } & \Gamma_{N}, & p & =p_{d} \quad \text { sur } \quad \Gamma_{D}
\end{array}
$$

où $\mathrm{K}$ est la perméabilité du milieu poreux, $\mu$ la viscosité du fluide, $\mathrm{p}$ la pression dans le fluide et, $\Gamma_{N}$ et $\Gamma_{D}$ sont les parties de $\Gamma$ où une condition de Neumann et une condition de Dirichlet sont imposées, $\Gamma=\Gamma_{N} \cup \Gamma_{D}$.

Dans la section qui suit on présente le schéma décentré amont d'ordre 1. Dans la section 3 on divise le domaine $\Omega$ en sous-domaines et on définit comment utiliser des pas de temps différents dans chaque sous-domaine. Enfin dans la section 4 on présente des résultats numériques.

\section{Discrétisation avec utilisation d'un pas de temps unique}

Dans la suite, pour simplifier les notations, on suppose que $\Omega=] a, b[\times] d, e[$ est un ouvert rectangulaire. Soit $\mathcal{T}_{h}$ une subdivision de $\Omega$ en rectangles $T_{j, k}: \Omega=\left(T_{j, k}\right)_{j, k}$. 
On discrétise l'intervalle $[a, b]$ en sous intervalles $\left[x_{j-1 / 2}, x_{j+1 / 2}\right], j=1, \ldots, N_{x}$ et l'intervalle $[d, e]$ en sous intervalles $\left[y_{k-1 / 2}, y_{k+1 / 2}\right], k=1, . ., N_{y}$ tels que

$$
\begin{array}{ll}
x_{1 / 2}=a \leq \ldots \leq x_{j} \leq \ldots \leq b=x_{N_{x}+1 / 2}, & h_{x}=x_{j+1 / 2}-x_{j-1 / 2} \\
y_{1 / 2}=c \leq \ldots \leq y_{k} \leq \ldots \leq d=y_{N_{y}+1 / 2}, & h_{y}=y_{k+1 / 2}-y_{k-1 / 2}
\end{array}
$$

Les notations utilisées pour la discrétisation en espace sont représentées sur la figure 1.

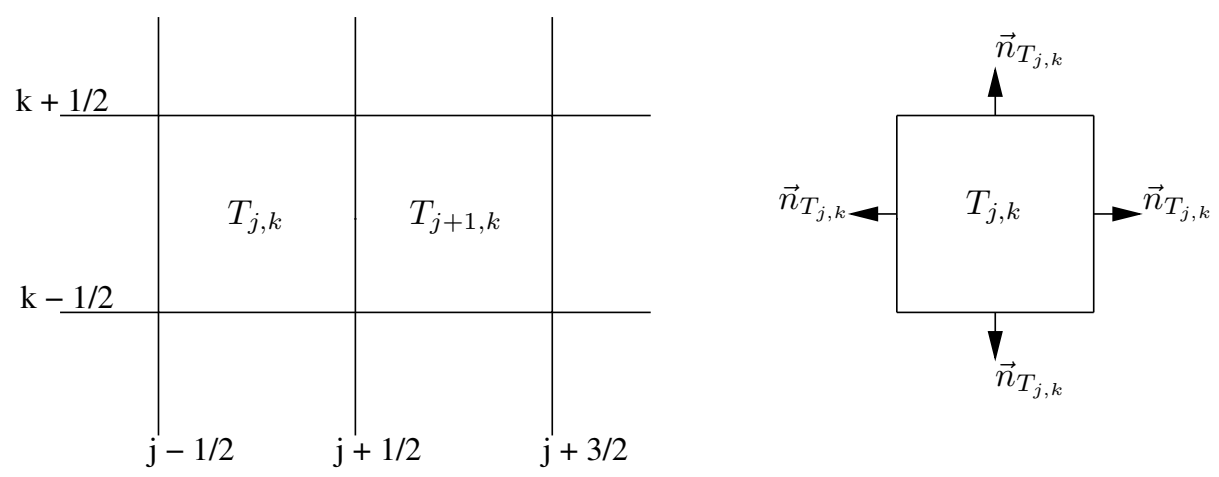

Figure 1. Notations utilisées pour les normales et les arêtes dans les deux mailles $T_{j, k}$ et $T_{j+1, k}$

L'intervalle de temps $[0, T]$ est discrétisé en $N_{t}$ sous intervalles $\left[t_{n}, t_{n+1}\right]$ tels que

$$
0=t_{0} \leq \ldots \leq t_{n} \leq \ldots \leq t_{N}=T, \quad \Delta t=t_{n+1}-t_{n} .
$$

On approche $c$ par une fonction constante par morceaux en temps et en espace $: c_{j, k}^{n}$ représente une approximation de la valeur moyenne de la concentration dans la maille $T_{j, k}$ à l'instant $n \Delta t . c^{n, *}$ est la valeur amont de la concentration sur chaque arête de la maille, que nous définissons un peu plus loin, $\vec{n}_{T_{j, k}}$ est la normale extérieure à la maille $T_{j, k}$.

Nous notons les normales aux arêtes verticales et horizontales respectivement

$$
\vec{n}_{x}=\left(\begin{array}{l}
1 \\
0
\end{array}\right), \quad \vec{n}_{y}=\left(\begin{array}{c}
0 \\
1
\end{array}\right) .
$$

Nous introduisons les débits d'eau à travers les arêtes de la discrétisation

$$
\begin{aligned}
& \left(Q_{x}\right)_{j+1 / 2, k}=\int_{A_{j+1 / 2, k}} \vec{u} \cdot \vec{n}_{x}, \quad 0 \leq j \leq N_{x}, \quad 1 \leq k \leq N_{y} \\
& \left(Q_{y}\right)_{j, k+1 / 2}=\int_{A_{j, k+1 / 2}} \vec{u} \cdot \vec{n}_{y}, \quad 1 \leq j \leq N_{x}, \quad 0 \leq k \leq N_{y},
\end{aligned}
$$


où $A_{j+1 / 2, k}$ est l'arête verticale interface entre les mailles $T_{j, k}$ et $T_{j+1, k}$, et $A_{j, k+1 / 2}$ est l'arête horizontale interface entre les mailles $T_{j, k}$ et $T_{j, k+1}$.

La concentration $c^{n, *}$ est la concentration amont définie par

$$
\begin{array}{rlrl}
c_{j+1 / 2, k}^{n, *}=c_{j, k}^{n} & \text { si } & & \left(Q_{x}\right)_{j+1 / 2, k} \geq 0 \\
c_{j+1 / 2, k}^{n, *}=c_{j+1, k}^{n} & & & \left(Q_{x}\right)_{j+1 / 2, k}<0 \\
c_{j, *}^{n, *} & =c_{j, k}^{n} \\
c_{j, k+1 / 2}^{n, *}=c_{j, k+1}^{n} & \text { si } & & \left(Q_{y}\right)_{j, k+1 / 2} \geq 0 \\
& & & \left(Q_{y}\right)_{j, k+1 / 2}<0 .
\end{array}
$$

Avec ces notations, le schéma d'Euler décentré amont explicite s'écrit

$$
\begin{aligned}
c_{j, k}^{n+1}=c_{j, k}^{n}-\frac{\Delta t}{h_{x} h_{y}} & {\left[\left(Q_{x}\right)_{j+1 / 2, k} c_{j+1 / 2, k}^{n, *}+\left(Q_{y}\right)_{j, k+1 / 2} c_{j, k+1 / 2}^{n, *}\right.} \\
- & \left.\left(Q_{x}\right)_{j-1 / 2, k} c_{j-1 / 2, k}^{n, *}-\left(Q_{y}\right)_{j, k-1 / 2} c_{j, k-1 / 2}^{n, *}\right] .
\end{aligned}
$$

et le schéma d'Euler décentré amont implicite s'écrit

$$
\begin{aligned}
& c_{j, k}^{n+1}+\frac{\Delta t}{h_{x} h_{y}} {\left[\left(Q_{x}\right)_{j+1 / 2, k} c_{j+1 / 2, k}^{n+1, *}+\left(Q_{y}\right)_{j, k+1 / 2} c_{j, k+1 / 2}^{n+1, *}\right.} \\
&\left.-\left(Q_{x}\right)_{j-1 / 2, k} c_{j-1 / 2, k}^{n+1, *}-\left(Q_{y}\right)_{j, k-1 / 2} c_{j, k-1 / 2}^{n+1, *}\right]=c_{j, k}^{n} .
\end{aligned}
$$

Pour les exemples qui vont suivre on va supposer avoir un champ de vitesse tel que $Q_{x} \cdot \vec{n}_{x}>0$ et $Q_{y} \cdot \vec{n}_{y}>0$, comme par exemple sur la figure 2 .

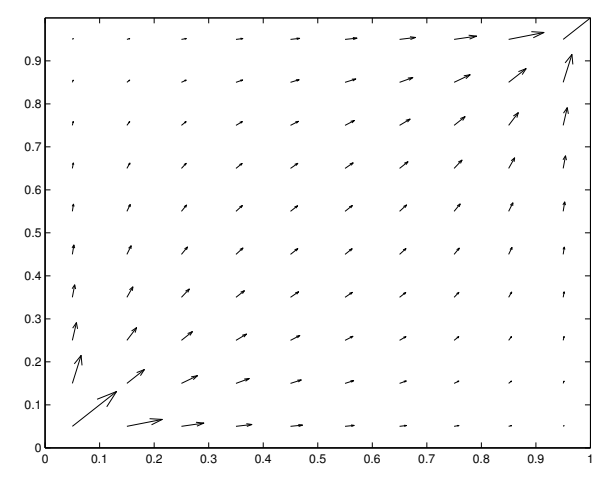

Figure 2. Vitesse de Darcy dans un domaine de perméabilité constante $\left(\vec{u} \cdot \vec{n}_{x}>0, \vec{u} \cdot \vec{n}_{y}>\right.$ $0)$

Alors les concentrations amont sont

$$
c_{j+1 / 2, k}^{n, *}=c_{j, k}^{n}, c_{j-1 / 2, k}^{n, *}=c_{j-1, k}^{n}, c_{j, k+1 / 2}^{n, *}=c_{j, k}^{n}, c_{j, k-1 / 2}^{n, *}=c_{j, k-1}^{n} .
$$


Dans ce cas le schéma explicite s'écrit maintenant

$$
\begin{aligned}
c_{j, k}^{n+1}= & c_{j, k}^{n}\left(1-\frac{\Delta t}{h_{x} h_{y}}\left(\left(Q_{x}\right)_{j+1 / 2, k}+\left(Q_{y}\right)_{j, k+1 / 2}\right)\right)+ \\
& c_{j-1, k}^{n} \frac{\Delta t}{h_{x} h_{y}}\left(Q_{x}\right)_{j-1 / 2, k}+c_{j, k-1}^{n} \frac{\Delta t}{h_{x} h_{y}}\left(Q_{y}\right)_{j, k-1 / 2},
\end{aligned}
$$

et le schéma implicite

$$
\begin{aligned}
& {\left[1+\frac{\Delta t}{h_{x} h_{y}}\left(\left(Q_{x}\right)_{j+1 / 2, k}+\left(Q_{y}\right)_{j, k+1 / 2}\right)\right] c_{j, k}^{n+1}} \\
& -\frac{\Delta t}{h_{x} h_{y}}\left(Q_{x}\right)_{j-1 / 2, k} c_{j-1, k}^{n+1}-\frac{\Delta t}{h_{x} h_{y}}\left(Q_{y}\right)_{j, k-1 / 2} c_{j, k-1}^{n+1}=c_{j, k}^{n} .
\end{aligned}
$$

Le schéma implicite est inconditionnellement stable mais le schéma explicite est stable si et seulement si les pas de discrétisation en temps et en espace satisfont la condition CFL[9]

$$
\frac{\Delta t}{h_{x} h_{y}} \leq \min _{1 \leq j \leq N_{x}, 1 \leq k \leq N_{y}} \frac{1}{\left[\left(Q_{y}\right)_{j, k-1 / 2}+\left(Q_{x}\right)_{j-1 / 2, k}\right]}
$$

La condition CFL est écrite sous cette forme inhabituelle car $\left(Q_{x}\right)$ et $\left(Q_{y}\right)$ sont ici des débits à travers les arêtes et non pas des flux.

\section{Utilisation de raffinement en temps par sous-domaines}

\subsection{Motivation}

Considérons maintenant un milieu poreux hétérogène caractérisé par une variation de perméabilité. Le domaine carré $\Omega$ est divisé en deux sous-domaines $\Omega_{1}$ et $\Omega_{2}$ de perméabilités $K_{1}$ et $K_{2}$ différentes (voir figure 3 ). 


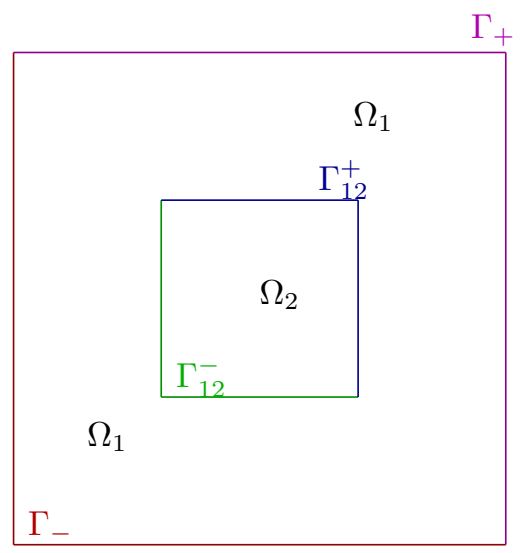

Figure 3. partition du domaine : définition des sous domaines et des interfaces : $\Gamma_{12}=$ $\Gamma_{12}^{+} \cup \Gamma_{12}^{-}$et $\Gamma=\Gamma_{+} \cup \Gamma_{-}$

On suppose que $K_{1} \geq K_{2}$. Dans un milieu à forte perméabilité le fluide a tendance à circuler rapidement, alors que dans un milieu peu perméable le fluide se déplace lentement. Dans la suite on va montrer comment utiliser des pas de temps $\Delta t_{l}, l=1,2$, différents dans chacun des sous-domaines $\Omega_{l}$, ces pas de temps étant déterminés par les conditions CFL associées à ces sous-domaines. La condition CFL implique que, pour une grille en espace donnée, le pas de temps est inversement proportionnel à la vitesse de Darcy.

Or on constate que la vitesse du fluide peut varier de façon importante : le fluide a tendance à aller plus vite quand il passe par une couche géologique de perméabilité importante et à aller moins vite lorsqu'il traverse une couche peu perméable. La figure 4 montre un exemple de variation de la vitesse en prenant $K_{1}=100$ et $K_{2}=1$. Dans cet exemple la vitesse de Darcy varie dans un rapport de 1 à 0.0035 .

Même dans le cas où le domaine est homogène, suivant le problème considéré, la vitesse peut varier de façon importante. Ainsi sur la figure 2 la vitesse varie dans un rapport de 1 à 0.0043 . Ceci est dû à la présence de grandes vitesses près de l'entrée et de la sortie du domaine.

L'objectif est en fait de découper le domaine en sous-domaines où la vitesse de Darcy ne varie pas de façon trop importante.

Notons néanmoins que les sous-domaines choisis de la sorte ne coïncident pas nécessairement avec les sous-domaines correspondant aux hétérogénéités. Ainsi, sur la figure 5, où nous avons inversé les valeurs de la perméabilité par rapport à la figure 4 , soit $K_{1}=1$ et $K_{2}=100$, les vitesses maximales dans $\Omega_{1}$ et $\Omega_{2}$ sont du même ordre de grandeur. 


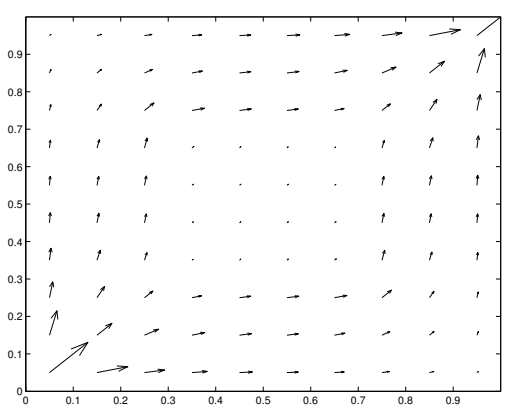

Figure 4. La vitesse du fluide dans le cas où $K_{1}=100$ et $K_{2}=1$

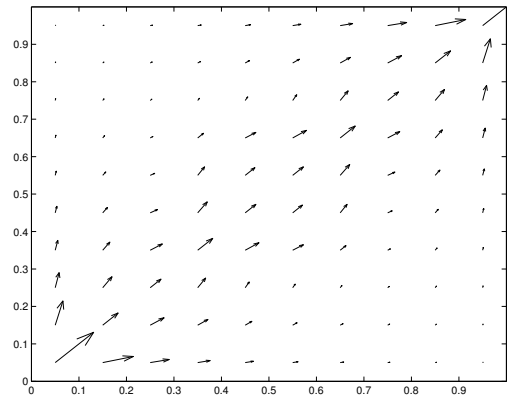

Figure 5. La vitesse du fluide dans le cas où $K_{1}=1$ et $K_{2}=100$

Revenons au cas où $K_{1} \geq K_{2}$ (cas de la figure 4 ). On évalue la condition CFL dans chaque sous-domaine :

$$
\frac{\Delta t_{l}}{h_{x} h_{y}} \leq \xi_{\ell}=\max _{\substack{1 \leq j \leq N_{x}, 1 \leq k \leq N_{y} \\ T_{j k} \subset \Omega_{\ell}}} \frac{1}{\left[\left(Q_{y}\right)_{j, k-1 / 2}+\left(Q_{x}\right)_{j-1 / 2, k}\right]}, \quad \ell=1,2 .
$$

On a évidemment $\xi_{2}>\xi_{1}$. On choisit des pas de temps par sous-domaine $\Delta t_{\ell}, \ell=1,2$ tels que la condition CFL soit vérifiée dans chaque sous-domaine et qu'ils soient dans un rapport entier. En conséquence on aura $\Delta t_{1}<\Delta t_{2}$.

On discrétise donc l'intervalle $[0, T]$ en sous-intervalles $I_{n}=\left[t_{n+1}, t_{n}\right], n=0, \ldots, N_{t}$ avec $N_{t} \in \mathbb{N}$ et on pose $\Delta t_{g}=\Delta t_{2}=t_{n+1}-t_{n}$. On calcule $M=E\left(\frac{\xi_{1}}{\xi_{2}}+1\right)$ où $E$ est la fonction partie entière et on pose $\Delta t_{f}=\Delta t_{1}=\frac{\Delta t_{g}}{M}$. Donc chaque intervalle $I_{n}$ est divisé en sous-intervalles $I_{n, m}=\left[t_{n, m}, t_{n, m+1}\right], m=0, \ldots, M$, tels que $t_{n, m}=t_{n}+\frac{m \Delta t_{2}}{M}$. Dans le sous-domaine 1 les pas de temps sont $\mathbf{M}$ fois plus petits que dans le sous-domaine 2. Ainsi on discrétise en temps l'équation en utilisant deux grilles différentes. Les inconnues et les pas de temps de chaque grille seront indicées par $\mathrm{f}$ et $\mathrm{g}$ (pour fin et grossier). On utilise ainsi les notations suivantes :

$$
\begin{array}{ll}
\left(c_{f}\right)_{j, k}^{n, m} \approx c\left(k h_{x}, j h_{y}, t_{n, m}\right) & \text { dans le sous-domaine } 1 \\
\left(c_{g}\right)_{j, k}^{n} \approx c\left(k h_{x}, j h_{y}, t_{n}\right) & \text { dans le sous-domaine } 2
\end{array}
$$


On pose $\lambda_{f}=\frac{\Delta t_{f}}{h_{x} h_{y}}$ et $\lambda_{g}=\frac{\Delta t_{g}}{h_{x} h_{y}}$. Dans le domaine 1 l'équation d'advection est approchée par

$$
\begin{aligned}
&\left(c_{f}\right)_{j, k}^{n, m+1}=\left(c_{f}\right)_{j, k}^{n, m}-\lambda_{f}[\left(c_{f}\right)_{j, k+1 / 2}^{n, m *}\left(Q_{y}\right)_{j, k+1 / 2}+\left(c_{f}\right)_{j+1 / 2, k}^{n, m *}\left(Q_{x}\right)_{j+1 / 2, k} \\
&\left.-\left(c_{f}\right)_{j, k-1 / 2}^{n, m *}\left(Q_{y}\right)_{j, k-1 / 2}-\left(c_{f}\right)_{j-1 / 2, k}^{n, m *}\left(Q_{x}\right)_{j-1 / 2, k}\right] .
\end{aligned}
$$

Sur les arêtes intérieures à $\Omega_{1}$ et sur celles incluses dans $\Gamma_{+}$on a

$$
\left(c_{f}\right)_{j+1 / 2, k}^{n, m *}=\left(c_{f}\right)_{j, k+1 / 2}^{n, m *}=\left(c_{f}\right)_{j, k}^{n, m} .
$$

Sur les arêtes incluses dans $\Gamma_{-}$on a $\left(c_{f}\right)_{j, 1 / 2}^{n, m *}=\left(c_{f}\right)_{1 / 2, k}^{n, m *}=1$.

A l'intérieur du domaine 2, le schéma décentré amont s'écrit :

$$
\begin{aligned}
&\left(c_{g}\right)_{j, k}^{n+1}=\left(c_{g}\right)_{j, k}^{n}-\lambda_{g}[\left(c_{g}\right)_{j, k+1 / 2}^{n *}\left(Q_{y}\right)_{j, k+1 / 2}+\left(c_{g}\right)_{j+1 / 2, k}^{n *}\left(Q_{x}\right)_{j+1 / 2, k} \\
&\left.-\left(c_{g}\right)_{j, k-1 / 2}^{n *}\left(Q_{y}\right)_{j, k-1 / 2}-\left(c_{g}\right)_{j-1 / 2, k}^{n *}\left(Q_{x}\right)_{j-1 / 2, k}\right] .
\end{aligned}
$$

Sur les arêtes intérieures à $\Omega_{2}$ on a $\left(c_{g}\right)_{j+1 / 2, k}^{n *}=\left(c_{g}\right)_{j, k+1 / 2}^{n *}=\left(c_{g}\right)_{j, k}^{n}$.

Reste maintenant à définir la concentration amont à l'interface entre 2 sous-domaines où des pas de temps différents sont utilisés. Deux situations se présentent alors : soit c'est à l'amont qu'on utilise des pas de temps petits (voir figure 6), soit c'est à l'aval qu'on utilise des pas de temps petits (voir figure 7).

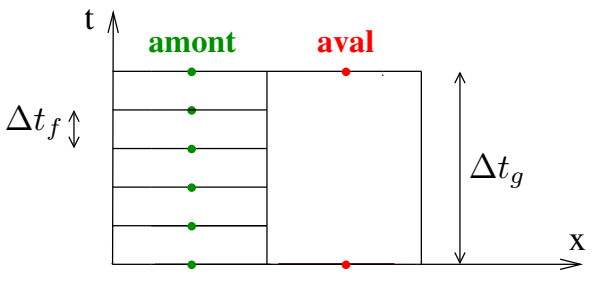

Figure 6. Discrétisation en temps sur $\Gamma_{12}^{-}(M=5)$.

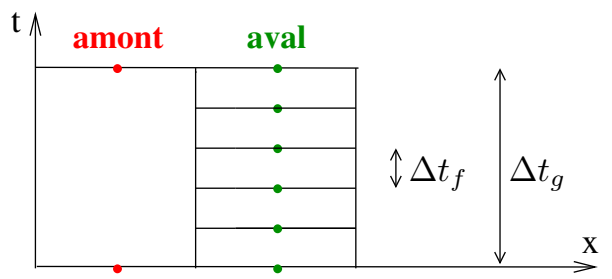

Figure 7. Discrétisation en temps sur $\Gamma_{12}^{+}(M=5)$.

Dans tous les cas il est nécessaire de faire des choix qui préserve la conservation du schéma et cet objectif justifie les formules ci-dessous :

\section{Cas des petits pas de temps à l'amont (figure 6)}

Schéma explicite : $\left(c_{g}\right)_{j+1 / 2, k}^{n, *}=\left(c_{g}\right)_{j, k+1 / 2}^{n, *}=\frac{1}{M} \sum_{m=0}^{M-1}\left(c_{f}\right)_{j, k}^{n, m}$.

Schéma implicite : $\left(c_{g}\right)_{j+1 / 2, k}^{n+1, *}=\left(c_{g}\right)_{j, k+1 / 2}^{n+1, *}=\frac{1}{M} \sum_{m=1}^{M}\left(c_{f}\right)_{j, k}^{n, m}$ 
Cas des grands pas de temps à l'amont (figure 7)

Schéma explicite : $\left(c_{f}\right)_{j+1 / 2, k}^{n, m, *}=\left(c_{f}\right)_{j, k+1 / 2}^{n, m, *}=\left(c_{g}\right)_{j, k}^{n}$.

Schéma implicite : $\left(c_{f}\right)_{j+1 / 2, k}^{n, m+1, *}=\left(c_{f}\right)_{j, k+1 / 2}^{n, m+1, *}=\left(c_{g}\right)_{j, k}^{n+1}$.

Dans le cas explicite, les pas de temps dans les différents sous-domaines sont choisis de sorte qu'ils soient les plus proches possible des maxima autorisés par les conditions CFL des différents sous-domaines. Dans le cas implicite, le pas de temps résulte d'un compromis précision-temps de calcul. On choisira donc des pas de temps qui sont autant que possible un multiple des pas de temps maximum autorisés par les conditions CFL des différents sous-domaines, ce multiple étant le même pour tous les sous-domaines.

\section{Les résultats numériques}

On présente maintenant des résultats numériques permettant de comparer la méthode avec un pas de temps par sous-domaine à celle où on utilise un pas de temps unique dans tout le domaine. Les comparaisons seront faites dans deux cas :

- le cas homogène, où la perméabilité est égale à 1 sur tout le domaine.

- le cas hétérogène où on a choisi $K_{1}=10$ dans $\Omega_{1}$ et $K_{2}=1$ dans $\Omega_{2}$. Le maillage du domaine $\Omega$ est supposé rectangulaire et uniforme. Il est constitué de carrés dont les côtés ont pour longueur h. La discrétisation est telle que les bords de $\Omega_{1}$ et $\Omega_{2}$ sont des réunions d'arêtes du maillage.

\subsection{Validation de la méthode en milieu homogène}

On présente sur la figure 8 à gauche les valeurs de la concentration sur chaque maille du domaine à l'instant $t=5$ calculées par la méthode utilisant un pas de temps $\Delta t_{1}=$ 0.45 sur le sous domaine 1 et un deuxième pas $\Delta t_{2}=2.35$ sur le sous domaine 2 , soit un rapport $M=5$. La figure 8 de droite montre des résultats calculés par la méthode utilisant un seul pas de temps $\Delta t=0.45$ pour tout $\Omega$. Visuellement les deux figures ne présentent pas de différence, ce qui est confirmé sur la figure 9 qui compare les fronts le long de la diagonale. On notera la diffusion numérique significative du schéma qui n'est que d'ordre 1 . 

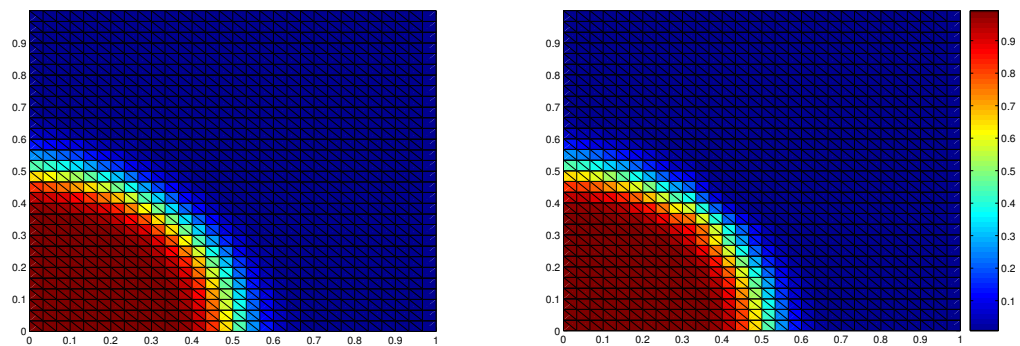

Figure 8. Concentrations dans le cas homogène $K=1$ calculées par la méthode utilisant un pas de temps par sous-domaine (à gauche) et par la méthode utilisant un seul pas de temps (à droite) à l'instant $t=30$

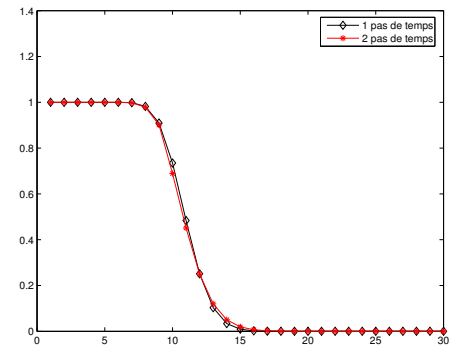

Figure 9. Comparaison des concentrations calculées le long de la diagonale du domaine à l'instant $t=30$ avec la méthode utilisant un pas de temps par sous-domaine $(\star)$ et celle utilisant un seul pas de temps unique $(\diamond)$.

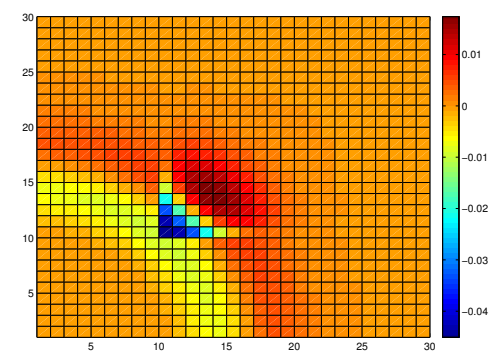

Figure 10. La différence des concentrations calculées par la méthode utilisant un pas de temps par sous-domaine et par la méthode utilisant un seul pas de temps à l'instant $t=30$ 
Sur la figure 10 on observe que la différence des concentrations calculées avec un pas de temps par sous-domaine et avec un seul pas de temps est faible, elle varie de 0 à 0.1 et elle est principalement localisée sur l'interface entre les deux sous-domaines.

\subsection{Validation de la méthode en milieu hétérogène}

Considérons d'abord le cas $K_{1}=10$ et $K_{2}=1$. La figure 11 montre l'évolution de la concentration aux instants respectifs $t=4, t=20, t=20$ et $t=30$. On observe comment les contaminants tendent à éviter la zone centrale où la perméabilité est faible. Dans ce calcul, compte tenu de la condition de courant 2, le pas de temps dans le domaine 1 est $\Delta t_{1}=0.045$ et dans le domaine $2 \Delta t_{2}=0.88$, soit un rapport $M=19$.
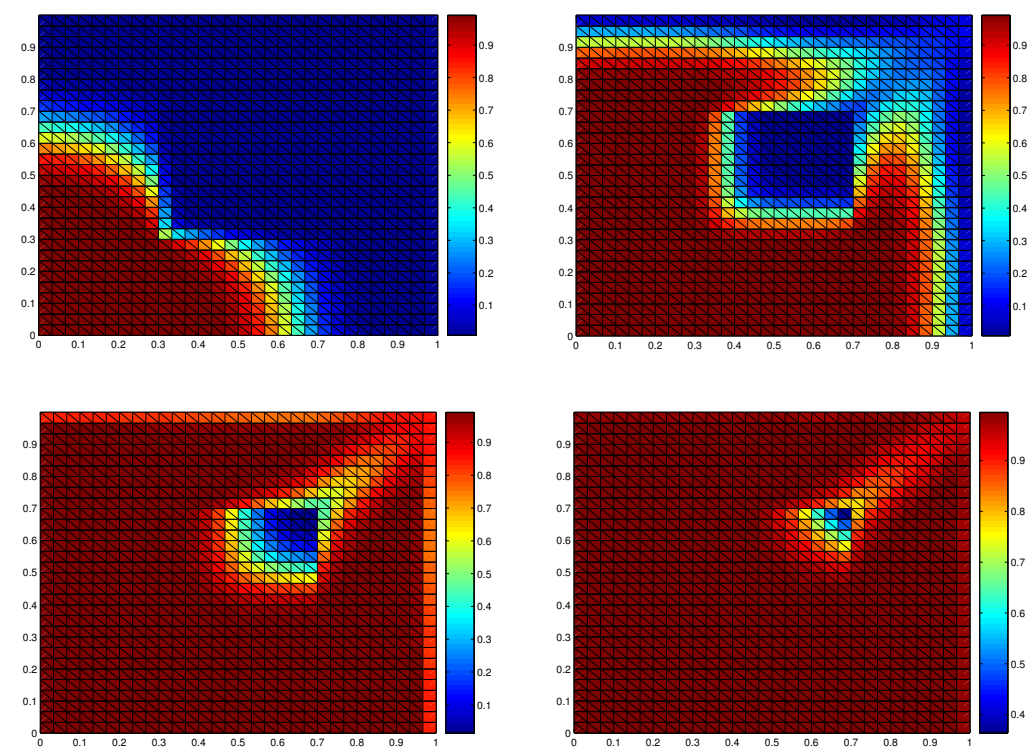

Figure 11. Concentrations dans le cas hétérogène $\left(K_{1}=10\right)$ calculées par la méthode utilisant un pas de temps par sous-domaines $t=4, t=10, t=20$ et $t=30$ avec $M=19$ 


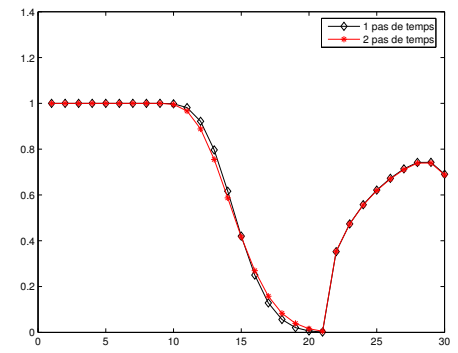

Figure 12. Comparaison des concentrations calculées le long de la diagonale du domaine à l'instant $t=15$ avec la méthode utilisant un pas de temps par sous-domaine $(\star)$ et celle utilisant un seul pas de temps unique $(\diamond)$.

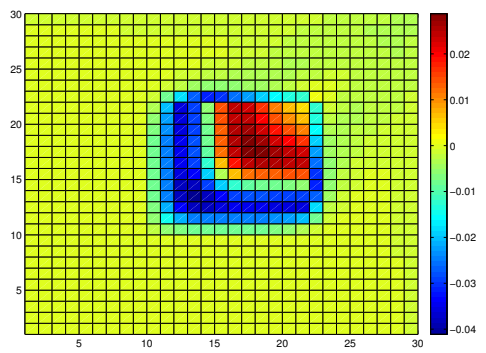

Figure 13. La différence des concentrations calculées par les deux méthodes sur chaque maille du domaine à l'instant $t=15$

A l'instant $t=15$ on compare le résultat obtenu avec celui obtenu en utilisant un pas de temps unique sur tout le domaine $\Delta t=0.045$. Les figures 12 et 13 montrent que les courbes sont proches et que la différence est petite.

On a aussi considéré le cas plus difficile où $K_{1}=100$, le champs de vitesse est celui représenté sur la figure 4.

La figure 14 montre l'évolution de la concentration aux instants $t=1$ et $t=3$. les pas de temps dans les deux sous domaines sont $\Delta t_{1}=0.0045$ et $\Delta t_{2}=0.65$ soit un rapport $M=144$.

Les figures 15 et 16 montrent que les résultats obtenus en comparant les deux méthodes sont très voisins. 

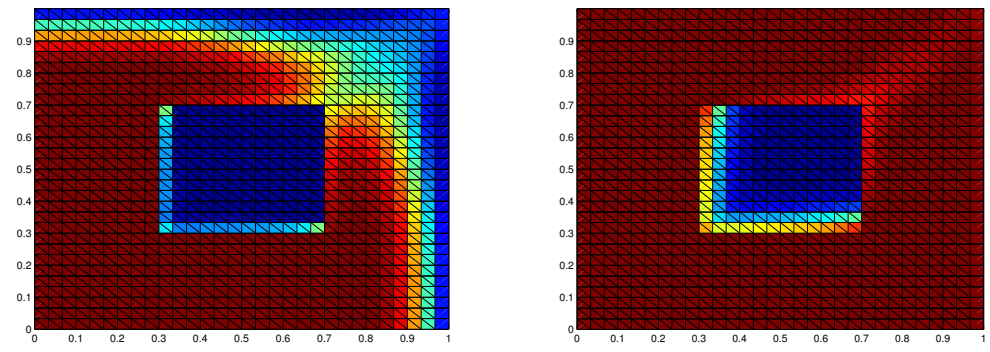

Figure 14. Concentrations dans le cas hétérogène $\left(K_{1}=100\right)$ calculées par la méthode utilisant un pas de temps par sous-domaine aux instants $t=1$ et $t=3$

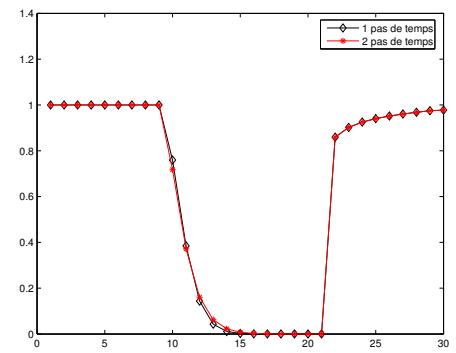

Figure 15. Comparaison des concentrations calculées le long de la diagonale du domaine à l'instant $t=3$ avec la méthode utilisant un pas de temps par sous-domaine ( $\star$ ) et celle calculée avec un pas de temps unique $(\diamond)$.

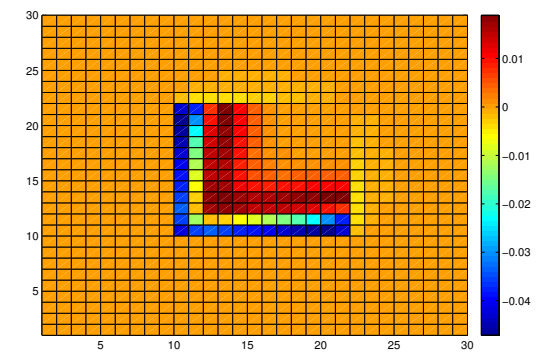

Figure 16. La différence des concentrations calculées par les deux méthodes sur chaque maille du domaine à l'instant $t=3$ 
Pour aller au delà des comparaisons visuelles on a construit les courbes de convergence des erreurs en norme $L^{1}$ et en norme $L^{2}$. Comme la solution exacte ne nous est pas connue, on a calculé l'erreur en prenant pour solution de référence la solution $c_{f}$ calculée sur un maillage très fin $h=1 / 640$. On a comparé cette solution avec celles calculées sur des maillages plus grossiers $h=1 / 10,1 / 20,1 / 40,1 / 80,1 / 160,1 / 320$. Sur ces grilles on a calculé les erreurs $L^{2}$ et $L^{1}$ à l'instant $t=10$ dans un cas hétérogène ( $\left.K_{1}=10, K_{2}=1\right)$ en utilisant la méthode utilisant un pas de temps par sous-domaine et celle calculée avec un pas de temps unique.

Les figures 17 montrent les erreurs $L^{2}$ et $L^{1}$ à l'échelle logarithmique calculée avec les deux méthodes. Les courbes d'erreurs des deux méthodes ne peuvent être distinguées. L'ordre $p_{2}$ de l'erreur $L^{2}$ calculé par les deux méthodes est compris entre $0.4827<p_{2}<$ 0.5280 . Tandis que l'ordre $p_{1}$ de l'erreur $L^{1}$ est compris entre $0.9565<p_{1}<1.0452$.
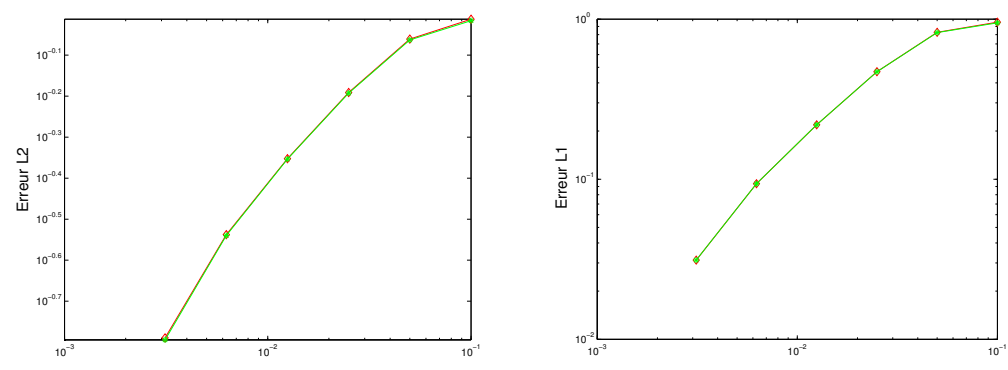

Figure 17. Erreurs $L 2$ (à gauche) et $L 1$ (à droite) en échelle logarithmique à $t=10 \mathrm{~s}$ entre la solution de référence calculée avec $h=1 / 640$ et les solutions calculées avec $h=1 / 10$, $1 / 20,1 / 40,1 / 80,1 / 160,1 / 320$ dans le cas hétérogène $K_{1}=10, K_{2}=1$ par la méthode utilisant un pas de temps par sous-domaine ( $\star$ ) et celle utilisant un seul pas de temps $(\diamond)$. Cas du schéma explicite.

Jusqu'ici nous n'avons montré aucun résultat concernant le schéma implicite. Cependant les comparaisons entre les résultats obtenus par les méthodes à un pas de temps par sous-domaine et à un pas de temps unique montrent encore qu'ils sont très voisins. Nous nous contentons ici, sur la figure 18, de montrer les courbes de convergence d'erreurs obtenues avec le schéma implicite en utilisant les mêmes pas de temps qu'avec le schéma explicite. De nouveau les courbes d'erreurs pour les deux méthodes ne peuvent être distinguées. 

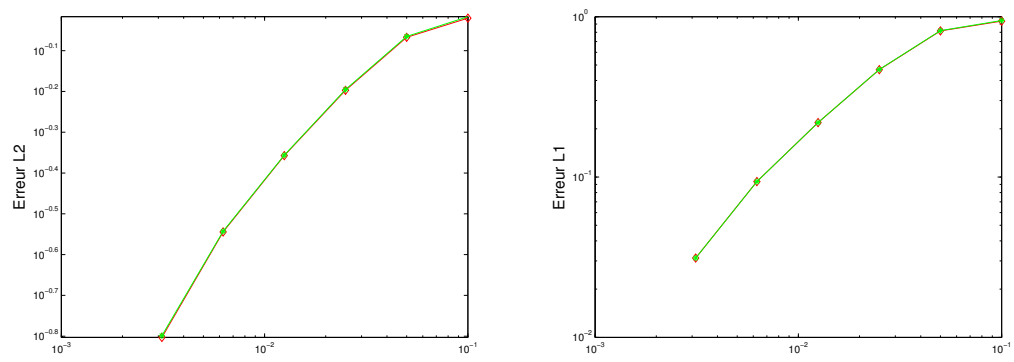

Figure 18. Erreurs $L 2$ (à gauche) et $L 1$ (à droite) en échelle logarithmique à $t=10 \mathrm{~s}$ entre la solution de référence calculée avec $h=1 / 640$ et la solution calculée avec $h=1 / 10$, $1 / 20,1 / 40,1 / 80,1 / 160$ et $1 / 320$ dans le cas hétérogène $K_{1}=10, K_{2}=1$ par la méthode utilisant un pas de temps par sous-domaine ( $\star$ ) et celle utilisant un seul pas de temps $(\diamond)$. Cas du schéma implicite.

\subsection{Conclusion}

Nous avons présenté une méthode de discrétisation en temps utilisant un pas de temps par sous-domaine pour résoudre une équation d'advection du premier ordre. Cette méthode, basée sur le schéma d'Euler, est conservatif et peut être utilisée aussi bien avec un schéma explicite qu'avec un schéma implicite. Dans les deux cas les résultats numériques ont montré qu'elle pouvait remplacer une méthode utilisant un pas de temps unique, sans perte de précision.

Ces résultats permettent d'espérer des gains de temps de calcul importants dans des situations complexes en 3 dimensions car ils ouvrent la possibilité d'adapter les pas de temps aux échelles de temps des sous-domaines, ces échelles peuvent varier de plusieur ordre de grandeur d'un milieu géologique à un autre.

\section{Bibliographie}

[1] Alain Bourgeat, Michel Kern, Stephan Schmacher and Jean Talandier. The COULPLEX test cases : Nuclear waste disposal Simulation. Computational Geosciences, 8(2) :83-98, 2004. Special Issue : Simulation of transport Around a Nuclear Waste Disposal Site : The Couplex Test Cases (Editors : Alain Bourgeat, Michel Kern).

[2] G. BAL, Y. MADAY. A parareal time discretization for non-linear PDE's with application to the pricing of an American put, Lect. Notes Comput. Sci. Eng. vol 23, Springer, Berlin 2002 MR1962689. 
[3] E. BÉcache, P. Joly, J. Rodriguez Space-time mesh refinement for elastodynamics. Numerical results, Comput. Methods Appl. Mech. Engrg, vol. 194, pp. 355-366, 2005.

[4] Clint Dawson, Robert Kirby , High resolution schemes for conservation laws with locally varying time steps, SIAM J. Sci. Comput., vol. 22, No. 6, pp. 2256-2281, 2001.

[5] M. Gander, L. Halpern, M. Kern A Schwarz Waveform Relaxation Method for Advection-Diffusion-Reaction Problems with Discontinuous Coefficients and non-Matching Grids. A apraître dans Domain Decomposition Methods in Science and Engineering, Series : Lecture Notes in Computational Science and Engineering, 2006.

[6] S. Piperno, C. FARhat, B. LARrouturou Partitioned procedures for the transient solution of coupled aeroelastic problems Part I : Model Problem, Theory, and Two-Dimensional Application, Comput. Meths. Appl. Mech. Engrg, vol. 124, No. 1-2, pp. 79-112, 1995.

[7] S. Piperno, C. FArhat Partitioned Procedures for the Transient Solution of Coupled Aeroelastic Problems Part II : Energy Transfer Analysis and Three-Dimensional Applications, Comput. Meths. Appl. Mech. Engrg, vol. 190, No. 24, pp. 3147-3170, 2001.

[8] P. Joly, J. Rodriguez A New Space-time Mesh Refinement Method for Elastodynamics, Proceedings of the 4th European Congress on Computational Methods in Applied Sciences and Engineering, Eccomas2004. Jyväskylä, Finland, July 24 - July 28, 2004.

[9] Randal J. LeVeque Finite Volume Methods for Hyperbolic Problems, Cambridge University Press, 2002.

[10] StAnley OSher AND Richard SANDERS Numerical Approximations to Nonlinear Conservation Laws With Locally Varying Time and Space Grids, Mathematics of computation, vol. 41, No. 164, pp. 321-336, 1983.

[11] ROBERT KIRBY , On the convergence of high resolution methods with multiple time scales for hyperbolic conservation laws, Mathematics of computation, vol. 72, No. 243, pp. 1239$1250,2002$. 